\title{
Exercises using the upper limbs hyperinflate COPD patients more than exercises using the lower limbs at the same metabolic demand
}

\author{
E.F. Porto1,2, A.A.M. Castro1,2, M. Velloso1,3, O. Nascimento1, \\ F. Dal Maso ${ }^{4}$, J.R. Jardim 1
}

ABSTRACT: Exercises using the upper limbs hyperinflate COPD patients more than exercises using the lower limbs at the same metabolic demand. E.F. Porto, A.A.M. Castro, M. Velloso, O. Nascimento, F. Dal Maso, J.R. Jardim.

Background. Lower and upper body exercise are mandatory constituents of a rehabilitation programme for patients with COPD. However, it is not known how much these exercises may induce pulmonary dynamic hyperinflation (DH).

Objective. To evaluate the $\mathrm{DH}$ in patients with COPD exercising the upper and lower parts of the body at the same metabolic demand.

Methods. Sixteen patients aged $63 \pm 13$ years and with a $\mathrm{FEV}_{1}$ of $1.5 \pm 0.7 \mathrm{~L}(41 \pm 11 \%$ pred $)$ were studied. Patients initially performed a maximal exercise test with the arms using the diagonal movement technique. The lower limbs were exercised on a treadmill at the same metabolic demand.
Results. Inspiratory capacity decreased $222 \pm 158 \mathrm{ml}$ $(9.8 \%)$ after the upper body exercise $(p<0.0001)$ and 148 $\pm 161 \mathrm{ml}(7 \%)$ after exercise with the lower body $(p=$ $0.0028)$ and a difference between the two groups was found $(p<0.05)$. There was no difference between resting IC before upper and lower limbs exercises $(p=0.8)$; increase in minute ventilation and in pulmonary ventilation in percentage of maximum voluntary ventilation and reduction of expiratory time were larger in the upper limbs exercise $(p<0.05)$. Dyspnea as measured by the Borg Scale was higher in the upper body $(3.9 \pm 2.2)$ than in the lower body $(2.3 \pm 1.3)$ at the end of the exercise $(p=0.033)$. Pulmonary ventilation and inspiratory capacity were correlated $(p=0.0001 ; r=0.82)$.

Conclusion. Exercise with the upper part of the body causes more DH and dyspnea than exercise with the lower part of the body at the same metabolic demand. Monaldi Arch Chest Dis 2009; 71: 1, 21-26.

1 Pulmonary Rehabilitation Center of Federal University of Sao Paulo (Unifesp),

2 Adventist University, São Paulo,

3 Federal University of Minas Gerais,

4 University of Erechim, RS., Brazil.

Correspondence: José R. Jardim, MD, Respiratory Diseases (Pneumologia - Unifesp), Rua Botucatu, 740 - 30 andar, 04023 062 São Paulo SP,Brazil; e-mail joserjardim@yahoo.com.br

\section{Introduction}

Patients with COPD present progressive difficulty in performing simple activities during their daily life (ADL) including those who require minimal effort such as elevation of the arms and simple domestic tasks. Those patients usually require periods of rest between tasks in order to accomplish them [1].

Although, approximately $80 \%$ of daily activities are performed using upper limbs [5], Velloso et al [2] have shown that simple daily tasks of daily living accomplished using upper limbs lead to the expenditure of approximately 50 to $60 \%$ of the maximum oxygen uptake and drive a high-minute ventilation reaching 60 to $70 \%$ of the maximum voluntary ventilation.

Dynamic hyperinflation is an important factor for effort limitation in patients with COPD [4-6]. In patients with COPD, mainly in moderate and se- vere stages, the shorter the expiratory time the more the air gets trapped, causing consequent hyperinflation and respiratory discomfort [3]. The dynamic hyperinflation phenomenon is classically demonstrated by measurement of pulmonary volumes in patients with COPD while performing physical activities and it has been well described for lower limbs.

In a recent study, Gigliotti et al [3] demonstrated that patients with COPD performing exercises on arm cycle ergometer developed dynamic lung hyperinflation, increased dyspnea and upper limbs fatigue. Apparently patients develop a lower level of dyspnea during lower limbs exercises than during upper limbs exercises. The physiological responses related to the degree of effort using the upper limbs are not yet totally understood but they may be related to a higher level of pulmonary ventilation and the development of dynamic hyperinflation $[3,4]$. 
Our objective was to assess pulmonary hyperinflation and ventilatory parameters in patients with COPD after performing an exercise using the upper limbs and compare them in the same patients after walking on a treadmill using the same metabolic load, in order to evaluate the influence of the ventilatory pattern on the dynamics of pulmonary hyperinflation.

\section{Methods}

This was a prospective study, evaluating patients with COPD who attended the COPD Unit at the Pulmonary Rehabilitation Center of the Federal University of São Paulo / Lar Escola São Francisco, Brazil. The study was approved by the Ethics Committee of the Federal University of São Paulo and all patients signed an informed consent form.

Inclusion criteria: Clinical diagnosis of COPD according to ATS/ERS criteria [7, 8]; clinical stability, no exacerbation during the previous 30 days; either gender; cognitive assessment by means of mini-mental testing with score above 24 points.

Exclusion criteria: Current smoker or nonsmoker of less than one year; severe co-morbidities such as heart disease, orthopedic or neurological diseases; extreme difficulty or inability to perform the inspiratory capacity maneuver; need for oxygen supplementation due to $\mathrm{SpO}_{2}<85 \%$ at rest or during exercise.

Protocol: At the preliminary visit they were submitted to a clinical examination to assure clinical stability and a spirometry was done pre and post-use of a bronchodilator (albuterol $400 \mathrm{mcg}$ ); forced vital capacity (FVC) and slow vital capacity (SVC) were measured (KoKo, USA), in accordance with methods recommended by ATS [7, 8].

At the second visit, the maximal inspiratory (PImax) and maximal expiratory (PEmax) pressures (Imbras ${ }^{\circledR} \pm 150 \mathrm{cmH}_{2} \mathrm{O}$, Brazil) [8] were measured and spirometry in the SVC mode was performed to obtain the inspiratory capacity (IC) according to the method discussed by Belmam et al [9]. Immediately afterwards, patients performed a maximal incremental upper limbs test (symptom limited) without the use of a bronchodilator with their expired gases being measured (K4b2 Cosmed $\left.^{\circledR}\right)$. Immediately after the test, IC, PImax and PEmax were measured again.

Patients rested for a minimal of 30 minutes or until arterial pressure, heart rate, inspiratory capacity, respiratory rate and dyspnea returned to basal values, they then performed an incremental test for lower limbs on a treadmill with measurement of exhaled gases until oxygen consumption had reached the same value obtained at the end of maximal upper limbs incremental test. Pre and post exercise patients had their IC, PImax and PEmax measured.

Inspiratory capacity measurement: Patients used a nasal clip and breathed through a mouthpiece connected to the circuit of the $\mathrm{KoKo}^{\circledR}$ equipment, which numerically and graphically recorded tidal volume values. When stability of the end expiratory volume was reached, the patient was asked to inspire up to total lung capacity. A minimum of three manoeuvers of up to a maximum of eight, were performed and, in order to be considered reproducible, two curves should not present a variation greater than $5 \%$ or $150 \mathrm{~mL}[10,12]$. Inspiratory capacity was registered as the value measured between the line of the end expiratory volume and total pulmonary capacity. The higher value of two reproducible curves was considered for analysis. Measurement of inspiratory capacity was done within two minutes after the tests.

Pulmonary hyperinflation: Pulmonary hyperinflation was considered when after the test there was a reduction in the inspiratory capacity of $10 \%$ and/or a reduction of more than $150 \mathrm{~mL}$, related to the basal value [11].

Unsupported arm exercise: The exercise consisted of lifting a halter Polypac ${ }^{\dagger}$ performing a diagonal movement based on the second diagonal of the proprioceptive neuromuscular facilitation technique [13] over the complete flexion-extension amplitude of the shoulder. This exercise starts with the arms crossed in the lap, with the elbows straight and the palms facing down; then the patient lifts the arms up, out and apart until fully extended above the head, approximately $45^{\circ}$ from the vertical, rotating the hand to finish with the palm facing posteriorly; a reverse movement is then done to return to the starting position [23]. The exercise continued alternating the arms till exhaustion. Initial weight was $250 \mathrm{~g}$ which was increased by $250 \mathrm{~g}$ each minute. The frequency and rhythm of the movements were imposed by a digital metronome (Qwiktime QT-3 $\$$ ) programmed to establish 20 repetitions per minute. Patients were encouraged to continue exercising until exhaustion, or occurrence of limiting symptoms (dyspnea and tiredness). The test was halted by the technician when: 1) The patient was observed to be using compensatory thoracic movements to accomplish the exercise; 2) The complete movement amplitude of the shoulder was not being reached; 3 ) or if the patient could no longer follow the metronome frequency.

Incremental exercise test with the legs: The test was performed on a treadmill (Lifestride model 7500, USA) following the Harbor protocol. During the first three minutes patients walked without no inclination on the treadmill at a fixed speed that they felt was comfortable, followed by a $1 \%$ increase in inclination each minute. Heart rate was continuously monitored at the CM5, AVF and V2 leads using electrocardiography (EP-3 Dixtal, Brazil) recordings being taken throughout the exercise and during the recovery period. The lower limbs incremental test was stopped when the oxygen consumption reached the same value as the maximal oxygen consumption reached in the maximal upper limbs incremental test.

Ventilatory and metabolic measurements: Ventilatory and metabolic variables were measured with the patient breathing through a mask, with air aliquots being constantly aspirated and 
measured by oxygen and carbon dioxide sensors (K4b2 Cosmed $\left.{ }^{\circledR}\right)$. Oxygen peripheral saturation $\left(\mathrm{SpO}_{2}\right)$ was continuously measured by means of a finger oximeter (Nonin, USA). Data on heart rate, $\mathrm{SpO}_{2}$, dyspnea and fatigue of the lower limbs were measured and recorded at minute intervals during the test.

Statistical analysis: Sample size was calculated based on the variability of the inspiratory capacity $(E / S)$ where $E / S$ is the magnitude of the expected effect divided by the standard deviation of the outcome variable with an error ' $\alpha$ ' of $5 \%$ and error ' $\beta$ ' of $20 \%$, according to the results from previous studies [14]. Sixteen patients were required to comply with the established research power requirements.

The data is expressed in mean and standard deviation. Analysis of variance with repeated measures was used; paired Student's $t$-test was used to assess the same variable pre and post-exercise. Whenever the variables did not follow normal distribution, the Mann-Whitney test was used to analyse differences among different groups; Pearson's correlation was used to establish correlations.

\section{Results}

Anthropometric and baseline pulmonary function data of the 16 patients is presented in table 1 . Four patients were using inhalatory corticosteroid regularly and only one patient had a hospitalisation record within the last year.

Upper limb exercise: The mean inspiratory capacity at rest was $2.35 \pm 0.44 \mathrm{~L}(2.11$ to $2.59 \mathrm{~L})$ and the median $2.34 \mathrm{~L}$ (figure 1). There was a mean reduction of $222 \pm 158 \mathrm{~mL}(9.8 \%)$ (69 to 370$)$ (median value $105 \mathrm{~mL}$ ) in the post-exercise inspiratory capacity with upper limbs $(p<0.0001)$, with 10 patients $(62.5 \%)$ complying with our definition of hyperinflation. Six of these patients presented a reduction in inspiratory capacity of at least $10 \%$, in addition to a loss greater than $150 \mathrm{~mL}$.

Lower limb exercise: The mean inspiratory capacity at rest was $2.34 \pm$ $0.47 \mathrm{~L}(2.10$ to $2.54 \mathrm{~L})$ and the median $2.34 \mathrm{~L}$ (figure 2). There was a mean reduction in the inspiratory capacity post-exercise on the treadmill of $148 \pm 161 \mathrm{~mL}$ $(7.2 \%) \quad(60$ to $237 \mathrm{~mL})$ (median value $80 \mathrm{~mL})(p=$ $0.0028)$, with only four patients $(25 \%)$ being hyperinflated.

Loss of inspiratory capacity was significantly higher after upper limbs exercise compared to lower limbs exercise at the same metabolic demand $(p$ $<0.05$ ) (figure 1). There was no difference between resting IC before upper and lower limb exercises $(p=0.8)$. The hyperinflation after the upper limbs exercise was higher than with the lower limbs exercise $(p<0.05)$.

Ventilatory pattern: During the upper limbs exercise, pulmonary ventilation and pulmonary ventilation in percentage of maximum voluntary ventilation were higher than those seen during the lower limbs exercise $(p<0.05)$ (table 2$)$. A reduction of expiratory time $(p<0.05)$ was only seen during the upper limbs exercise. There was a strong correlation between the increase in pulmonary ventilation and the decrease in the inspiratory capacity during the exercise with upper limbs $(r=0.82 ; p<0.0001)$ (figure 2$)$.

Table 1. - Subject characteristics

\begin{tabular}{lc}
\hline Variables & Mean \pm SD \\
\hline Age $($ years $)$ & $63.1 \pm 13.2$ \\
Height $(\mathrm{cm})$ & $164.1 \pm 13.1$ \\
BMI $\left(\mathrm{kg} / \mathrm{m}^{2}\right)$ & $25.9 \pm 6.0$ \\
$\mathrm{FVC}(\%$ predicted) before bronchodilator & $69.4 \pm 14.4$ \\
$\mathrm{FEV}_{1}(\%$ predicted) before bronchodilator & $36.0 \pm 10.5$ \\
$\mathrm{FEV}_{1} / \mathrm{FVC}$ before bronchodilator & $0.44 \pm 0.07$ \\
$\mathrm{FVC} \%$ predicted) after bronchodilator & $74.0 \pm 13.0$ \\
$\mathrm{FEV}_{1}(\%$ predicted $)$ after bronchodilator & $41.4 \pm 11.2$ \\
$\mathrm{FEV}_{1} / \mathrm{FVC}$ after bronchodilator & $0.46 \pm 0.01$ \\
$\Delta \mathrm{FEV}_{1}(\mathrm{ml})$ before and after bronchodilator & $50.0 \pm 16.0$ \\
$\Delta \mathrm{FVC}(\mathrm{ml})$ before and after bronchodilator & $180.0 \pm 99.0$
\end{tabular}

\# BMI = body mass index; FVC (\%pred. $)=$ forced vital capacity $\%$ predicted; $\mathrm{FEV}_{1}(\%$ pred. $)=$ forced expiratory volume in the first second $\%$ predicted; $\mathrm{FEV}_{1} / \mathrm{FVC}=$ forced expiratory volume in the first second/ forced vital capacity ratio, $\Delta \mathrm{FEV}_{1}(\mathrm{ml})=$ forced expiratory volume in the first second delta (post - pre bronchodilator) in milliliters; $\triangle \mathrm{FVC}(\mathrm{ml})=$ forced vital capacity delta (post pre bronchodilator) in milliliters.

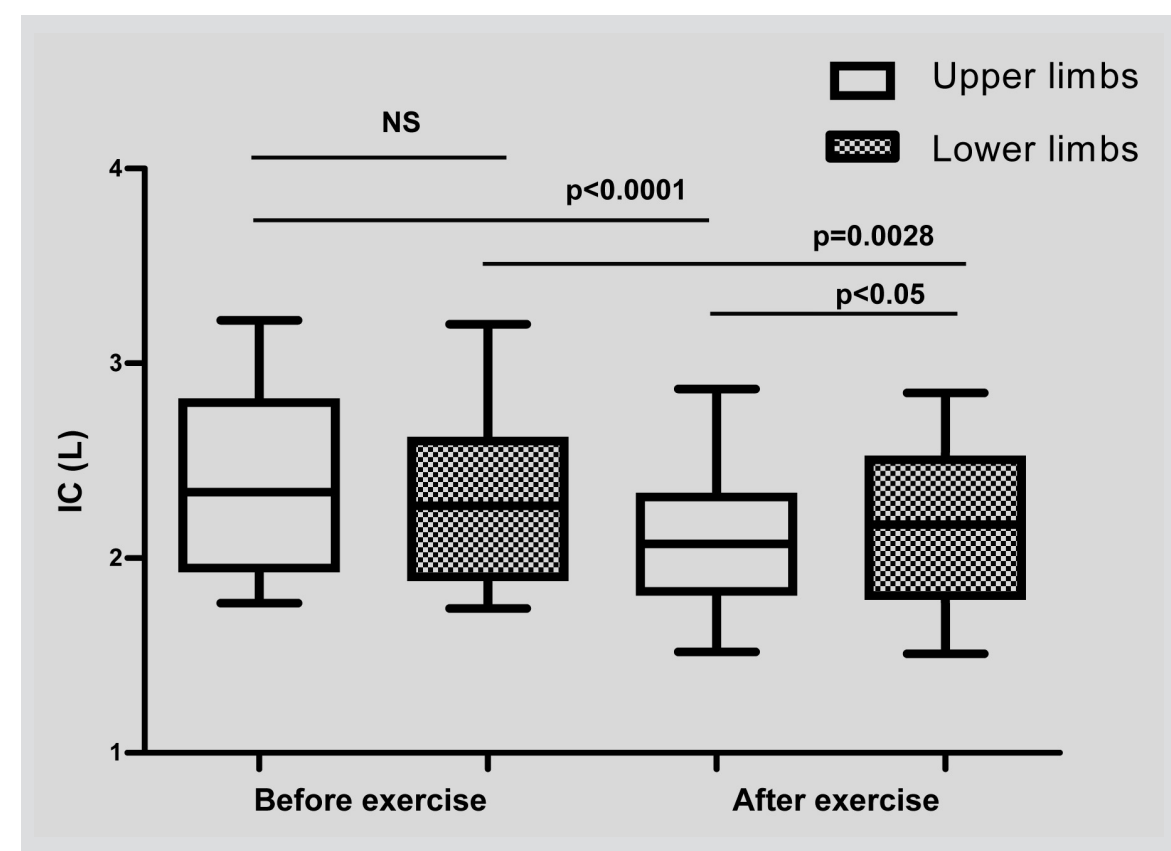

Fig. 1. - Inspiratory capacity pre and post incremental test of upper and lower limbs. IC = inspiratory capacity. 


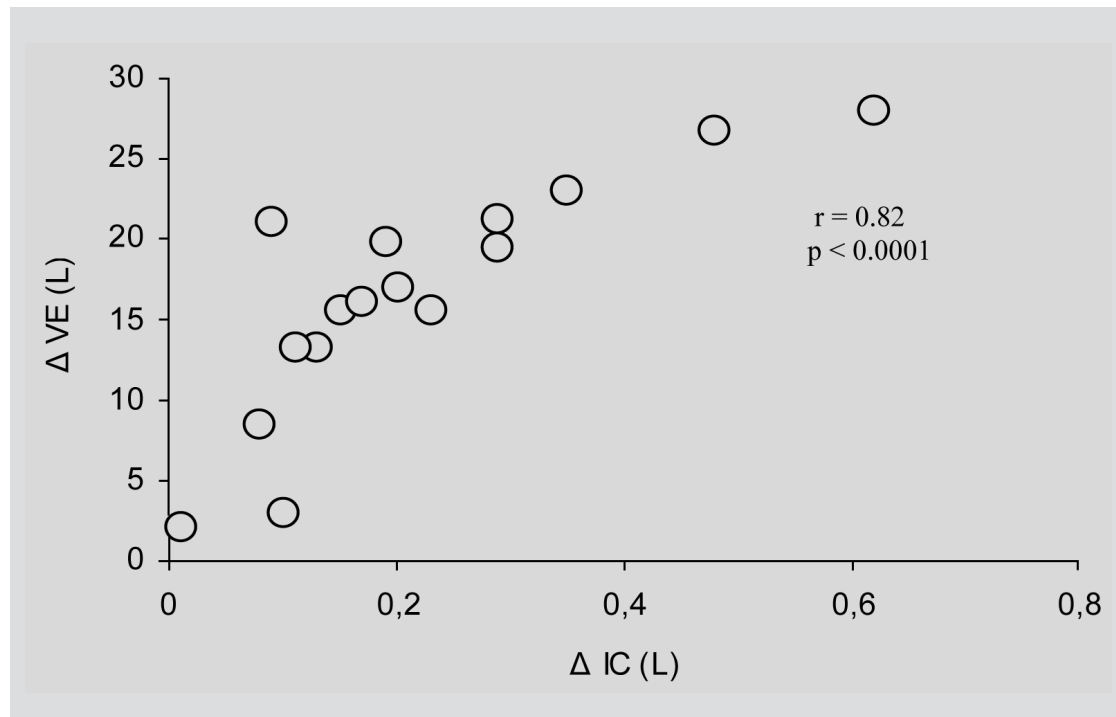

Fig. 2. - Correlation between increase in pulmonary ventilation and decrease of inspiratory capacity after upper limbs exercise test. $\Delta \mathrm{IC}=$ inspiratory capacity delta (post-pre exercise); $\Delta \mathrm{VE}=$ pulmonary ventilation delta (post-pre exercise).

\section{Discussion}

The major finding in this study was the observation that approximately $60 \%$ of COPD patients developed dynamic hyperinflation during the upper limbs exercises. A smaller number of patients hyperinflated doing lower limbs exercises at the same oxygen consumption.

The second diagonal of the proprioceptive neuromuscular facilitation technique was used for exercising the upper limbs as it recruits a larger number of muscles than the exercise performed on an arm cyclergometer. It is also believed that this technique is more functional than other techniques due to its similarity to the activities of daily living arm movements. Diagonal exercises have been used for upper limbs training [16, 17, 22].
There is no doubt that the exercise protocols for the upper and lower limbs differ but our main purpose was to compare two common kinds of exercise for these limbs at the same metabolic demand.

Gigliotti et al [3] had already shown that upper limbs exercises with an arm cycle ergometer may hyperinflate COPD patients. But exercises with diagonal movements are more realistic to the activities of daily living accomplished by COPD patients with upper limbs in real life. No study has ever before evaluated dynamic hyperinflation using the diagonal technique.

We found a strong association of dynamic hyperinflation and pulmonary ventilation enhancement by shortening the expiratory time in our COPD patients leading to shallow breathing (figure 2). Few studies have examined the ventilatory response during activities with upper limbs. Tangri and Wolf [15] were among the first investigators to study the ventilatory patterns of patients with COPD performing daily routine activities using the upper limbs. They observed that simple tasks, such as combing their hair and putting on their shoes led to a precipitated, superficial and irregular respiratory pattern. More recently, other studies have shown that the simple elevation of the arms by patients with COPD reduced the vital capacity and at the same time increased $\mathrm{O}_{2}$ consumption and pulmonary ventilation by $16 \%$ and $24 \%$, respectively $[16,17]$. Despite the fact that inspiratory capacity was not

Table 2. - Ventilatory pattern at rest and peak exercise of upper and lower limbs

\begin{tabular}{lcccc}
\hline & \multicolumn{2}{c}{ Exercise of upper limbs } & \multicolumn{2}{c}{ Exercise of lower limbs } \\
\hline Variables & Rest & Peak & Rest & Peak \\
\hline $\mathrm{VO}_{2} / \mathrm{ml} / \mathrm{kg} / \mathrm{min}$ & $3.5 \pm 0.8$ & $9.3 \pm 2.7$ & $3.7 \pm 0.6$ & $9.7 \pm 2.8$ \\
$\mathrm{VE}(\mathrm{L})$ & $14.1 \pm 5.1$ & $31.0 \pm 7.4 *$ & $14.2 \pm 5.3$ & $24.0 \pm 6.3$ \\
$\mathrm{VD} / \mathrm{VT}$ & $0.4 \pm 0.13$ & $0.5 \pm 0.11$ & $0.3 \pm 0.14$ & $0.3 \pm 0.10$ \\
$\mathrm{f}$ (breaths/min) & $21.0 \pm 6$ & $26.0 \pm 6$ & $21.0 \pm 5$ & $25.0 \pm 7$ \\
$\mathrm{TI}(\mathrm{sec})$ & $1.1 \pm 0.23$ & $0.9 \pm 0.14$ & $1.0 \pm 0.20$ & $0.9 \pm 0.17$ \\
$\mathrm{TE}$ (sec) & $1.7 \pm 0.21$ & $1.4 \pm 0.22 *$ & $1.9 \pm 0.12$ & $1.6 \pm 0.11$ \\
$\mathrm{TI} / \mathrm{TTOT}$ & $0.4 \pm 0.10$ & $0.48 \pm 0.12$ & $0.48 \pm 0.10$ & $0.45 \pm 0.11$ \\
VT/TI (mL/sec.) & $0.63 \pm 0.13$ & $1.1 \pm 0.21$ & $0.70 \pm 0.16$ & $1.1 \pm 0.12$ \\
VT (L) & $0.70 \pm, 012$ & $1.0 \pm 0.23$ & $0.70 \pm 0.13$ & $1.0 \pm 0.33$ \\
VE/MVV & $0.23 \pm 0.13$ & $0.53 \pm 0.15^{*}$ & $0.23 \pm 0.13$ & $0.41 \pm 0.17$ \\
Dyspnea ratings (Borg) & $0.5 \pm 0.1$ & $3.9 \pm 2.2^{*}$ & $0.5 \pm 0.1$ & $2.3 \pm 1.3$ \\
\hline
\end{tabular}

$* \mathrm{p}<0.05$ peak upper limbs vs peak lower limbs.

$\# \mathrm{VO}_{2}=$ oxygen consumption; $\mathrm{VE}=$ pulmonary ventilation; $\mathrm{VD} / \mathrm{VT}=$ dead space/ tidal volume ratio; $\mathrm{f}=$ respiratory rate; $\mathrm{TI}=$ inspiratory time; TE $=$ expiratory time TI/TTTOT $=$ inspiratory time/ total respiratory time ratio; $\mathrm{VT} / \mathrm{TI}=$ mean inspiratory flow; $\mathrm{VT}$ = tidal volume; $\mathrm{VE} / \mathrm{MVV}=$ pulmonary ventilation/maximum ventilatory ventilation ratio. 
measured by these authors it is conceivable to admit that the decrease in vital capacity may be attributed to dynamic hyperinflation.

Lake et al [18] studied the ventilatory pattern of patients with COPD maximally exercising in an arm cyclergometer and found: an increase in pulmonary ventilation, respiratory rate and $\mathrm{T}_{\mathrm{I}} / \mathrm{T}_{\mathrm{TOT}}$ ratio and also a reduction in the expiratory time. They also observed that the greater the neuromuscular drive, as measured by the mean inspiratory airflow, the greater the level of dyspnea.

Our results at the peak of the upper limbs exercise are similar to those observed by Lake et al [18] There was an increase in pulmonary ventilation, respiratory rate, tidal volume to inspiratory time ratio $\left(\mathrm{V}_{\mathrm{T}} / \mathrm{T}_{\mathrm{I}}\right)$, dead space to tidal volume ratio $\left(\mathrm{V}_{\mathrm{D}} / \mathrm{V}_{\mathrm{T}}\right)$ and dyspnea; a reduction in the expiratory $\left(\mathrm{T}_{\mathrm{E}}\right)$ and inspiratory $\left(\mathrm{T}_{\mathrm{I}}\right)$ times, with maintenance of the inspiratory time to total time ratio $\left(\mathrm{T}_{\mathrm{I}}\right.$ / $\mathrm{T}_{\mathrm{TOT}}$ ) occurred concomitantly. These results clearly show that an alteration in the ventilatory pattern occurred while the COPD patients were performing the exercise using their upper limbs.

In patients with COPD, exercise limitation is predominantly associated with high ventilation. In our study, pulmonary ventilation at peak exercise corresponded to $74 \%$ of maximal voluntary ventilation. This elevated percentage is significantly higher than the ventilation reached by healthy individuals when performing a maximal cardiopulmonary exercise (usually 50-60\% of their maximal voluntary ventilation). High ventilation related to maximal voluntary ventilation was also found by Velloso et al [2, 19] while assessing patients with COPD performing simple daily activities using the upper limbs.

We observed that there was a greater hyperinflation following the exercise performed using the upper limbs than in the one performed using the lower limbs, at the same $\mathrm{VO}_{2}$. Similarly, the exercise using the upper limbs led to greater pulmonary ventilation, higher respiratory rate, dyspnea, pulmonary ventilation in percentage of maximal voluntary ventilation and dead space/tidal volume ratio, as well as a shortening of the expiratory and inspiratory times. The slight increase in the mean inspiratory flow during both exercises reflects an increase in the neuromuscular drive. Other studies have looked for an explanation for the higher ventilation during arm exercises. Lind et al [20] showed that during a handgrip exercise the increased muscle tension reduces the blood flow to the muscles of the forearm and that this reduction is inversely correlated to an increase in pulmonary ventilation. Furthermore, Bevegard et al [21] showed that blood flow reduction during an arm sustentation exercise occurs because of adrenergic output and vasoconstriction; the authors concluded that there is a more intense reduction of blood flow to smaller muscle groups, such as in the forearm compared to the lower limbs exercise, leading to higher blood lactate accumulation with an increased pulmonary ventilation stimulation. The proportional greater reduction in the forearm blood circulation and increase in blood lactate may be a possible mechanism that caused our patients to present greater pulmonary ventilation during the arm exercise compared to the treadmill exercise. Usually the greater pulmonary ventilation that is adopted by individuals when performing an exercise includes a shortening of inspiratory and expiratory times. However, the shortening of expiratory time in COPD patients usually causes air trapping and hyperinflation.

A possible limitation to our study is the fact that we did not measure the static lung volume of our patients, such as residual volume and total lung capacity. This measurement would probably have revealed additional data on the respiratory status of our subjects. However our focus was on the evaluation of dynamic lung changes and the method applied could answer our question. As this is the first study comparing pulmonary hyperinflation in patients with COPD in exercises using both upper and lower limbs at the same metabolic load, it is difficult to compare our results to other studies.

We conclude that a substantial number of COPD patients, when performing movements with upper limbs, similar to the ones often used in daily activities, may hyperinflate. Hyperinflation generated by an exercise using the upper limbs is more severe than that generated by lower limbs exercises when performed at the same metabolic load due to shallow breathing. We suggest that further studies should be done regarding the association between pulmonary hyperinflation and the duration and load imposed on the upper limbs during exercises in COPD patients.

\section{References}

1. Leidy NK. Psychometric properties of the functional performance inventory in patients with chronic obstructive pulmonary disease. Nurs Res 1999; 48: 20-28.

2. Velloso M, Stella SG, Cendon S, et al. Metabolic and ventilatory parameters of four activities of daily living accomplished with arms in COPD patients. Chest 2003; 123: 1047-1053.

3. Gigliotti, F, Coli C, Bianchi R, et al. Arm exercise and hyperinflation in patients with COPD. Effect of arm training. Chest 2005; 18: 125-132.

4. O'Donnell DE, Lam M, Webb KA. Measurement of symptoms, lung hyperinflatrion, and endurence during exercise in chronic obstructive pulmonary disease. Am J Respir Crit Care Med 1998; 159: 720-727.

5. Potter WA, Olafsson S, Hyatt RE. Ventilatory mechanics and expiratory flow limitation during exercise in patients with obstructive lung disease. J Clin Invest 1971; 50: 910-919.

6. Couser JI, Martinez FJ, Celli BR. Respiratory response and ventilatory muscle recruitment during arm elevation in normal subjects. Chest 1992; 101: 336-340.

7. American Thoracic Society. Standards for the diagnosis and care of patients with chronic pulmonary obstructive disease. Am J Respir Crit Care Med 1995; 52: 77-120.

8. American Thoracic Society. Lung function testing: selection of reference values and interpretative strategies. Am Rev Respir Dis 1995; 144: 1202-24.

9. Belmam MJ, Botinick WC, Shin J. Inhaled bronchodilators reduce dynamic hyperinflation during exercise in patients with chronic obstructive pul- 
monary disease. Am J Respir Crit Care Med 1996; 153: 967-975.

10. Neder JÁ, Adreonni S, Lerario MC, et al. Reference values for lung function test. II. Maximal respiratory pressure and voluntary ventilation. Braz J Med Biol Res 1999; 32: 719-27.

11. Yan S, Dariusz K., Sliwinsk P. Reliability of inspiratory capacity for estimating end - expiratory lung volume changes during exercise in patients with chronic obstructive pulmonary disease. Am J Respir Crit Care Med 1997; 156: 55-59.

12. O'Donnell DE, Revil SM Webb KA. Dynamic hyperinflation and exercise intolerance in chronic obstructive pulmonary disease. Am J Respir Crit Care Med 2001; 164: 770-777.

13. Shimura K, Kasai T. Effects of proprioceptive neuromuscular facilitation on the initiation of voluntary movement and motor evoked potentials in upper limb muscles. Human Movement Science 2002; 101-113.

14. Hulley, Stephen D. Delineando a Pesquisa Clínica: Uma Abordagem Epidemiológica. $2^{\circ}$ Ed. Art-Med 2003; Porto Alegre-RS.

15. Tangri S, Woolf CR. The breathing pattern in chronic obstructive lung disease during the performance of some common daily activities. Chest 1973; 63: 126-129.

16. Scott KE, James W, Stephen T, et al. Ventilatory re- sponse to arm elevation. Am J Respir Crit Care Med 1995; 152: 211-216.

17. Fernando JM, Robert LS, Kevin RF, et al. Respiratory response during arm elevation in isolated diaphragm weakness. Am J Respir Crit Care Med 1999; 160: 480-486.

18. Lake Fr, Handerson K, Briffa T, et al. Upper limbs and lower limbs exercise training in patients with chronic air-flow obstruction. Chest 1990; 97: 1077-84.

19. Velloso M, Jardim JR. Study of energy expenditure during activities of daily living using and not using body position recommended by energy conservation techniques in patients with COPD. Chest 2006; 130; 126-132.

20. Lind AR, Taylor SH, Humphreys BM, et al. The circulatory effects of sustained voluntary muscle contraction. Clin Sci 1964; 27: 229-244.

21. Bevegard S, Freyschuss U, Strandell T. Circulatory adaptation to arm and leg exercise in supine sitting position. J Appl Physiol 1968; 25: 528-532.

22. Ries AL, Ellis B, Hawkins RWH. Upper extremity exercise training in chronic obstructive pulmonary disease. Chest 1988; 93: 688-92.

23. Bauldoff GS, Hoffman LA, Sciurba F, Zullo TG. Home-based, upper arm exercise training for patients with chronic obstructive pulmonary disease. Heart \& Lung 1996; 25: 288-94.

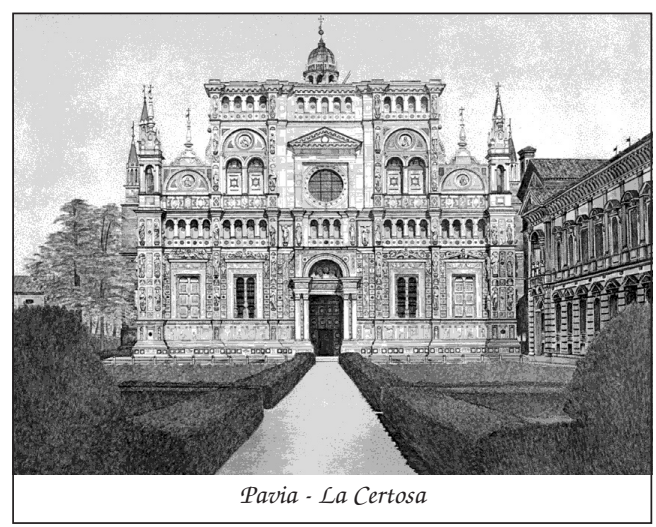

